\title{
TOMA DE DECISIONES FRENTE A DOLOR REFRACTARIO EN PACIENTE ONCOLÓGICA JOVEN
}

\author{
DECISION MAKING ON REFRACTORY PAIN IN A YOUNG \\ ONCOLOGICAL PATIENT
}

\section{DECISÃO FAZENDO CONTRA A DOR REFRATÁRIA NO PACIENTE ONCOLÓGICO}

\author{
Héctor de los Santos \\ Universidad Católica del Uruguay, Montevideo, Uruguay \\ hdls1967@gmail.com \\ ORCID 0000-0001-6959-9814

\section{Bettina Frugoni} \\ Universidad Católica del Uruguay, Montevideo, Uruguay \\ ORCID 0000-0003-1427-3061
}

\author{
Ana Guedes \\ Universidad de la República, Montevideo, Uruguay \\ ORCID 0000-0002-3373-825
}

\author{
Adriana Redin \\ Universidad Católica del Uruguay, Montevideo, Uruguay \\ ORCID 0000-0002-4888-957X
}

DOI: https://doi.org/10.22235/ech.v8i1.1796

Recibido: $14 / 10 / 2018$

Aceptado: 10/03/2018

\section{RESUMEN}

La gran mayoría de los pacientes oncológicos en etapas avanzadas presentan dolor. La mayoría de éstos son controlados con analgésicos siguiendo la escalera analgésica de la Organización Mundial de la Salud. Sin embargo, un porcentaje de pacientes persisten con dolor. Estos son pacientes con dolor de difícil control que se benefician muchas veces de medidas intervencionistas. Un porcentaje aún menor de pacientes mantiene el dolor como síntoma refractario a pesar de las medidas terapéuticas adecuadas. En estos casos está indicada la sedación paliativa. Se presenta aquí el caso de una paciente joven con dolor refractario que requirió sedación paliativa, en una unidad de cuidados paliativos de una institución privada de Montevideo.

Palabras clave: Dolor, gestión clínica, cuidados paliativos, sedación.

\section{ABSTRACT}

The vast majority of cancer patients in advanced stages feel pain. Most are controlled with analgesics following the WHO analgesic ladder. However, a percentage of patients persist feeling pain. These are patients with difficult-to-control pain who often benefit from 
interventional measures. An even smaller percentage of patients keep on feeling pain as a refractory symptom despite adequate therapeutic measures. In these cases, palliative sedation is indicated. We present the case of a young patient with refractory pain who required palliative sedation in a palliative care unit of a private institution in Montevideo.

Keywords: Pain, clinical management, palliative care, sedation.

\section{RESUMO}

A grande maioria dos pacientes com câncer em estágios avançados têm dor. A maioria destas dores são controladas com analgésicos, de acordo com a escada analgésica da OMS. No entanto, uma porcentagem de pacientes continua sentindo dores. Estes são pacientes com dor de difícil controle que frequentemente se beneficiam de medidas de intervenção. Uma porcentagem ainda menor de pacientes mantêm a dor como um sintoma refratário, apesar das medidas terapêuticas adequadas. Nestes casos, a sedação paliativa é indicada. Apresentamos o caso de uma paciente jovem com dor refratária que precisou de sedação paliativa em uma unidade de cuidados paliativos de uma instituição privada em Montevidéu.

Palavras-chave: Dor, manejo clínico, cuidados paliativos, sedação.

\section{INTRODUCCIÓN}

El dolor, de acuerdo con la International Association for Study of Pain (IASP) se define como "experiencia sensorial y emocional desagradable asociada, o no, a daño tisular real o potencial descrita en función de dicho daño" (1). El dolor tiene un gran impacto sobre la calidad de vida y un efecto devastador en el enfermo terminal. Cuando no es aliviado, es capaz de convertirse en el centro de la vida de la persona. El dolor total afecta a la integridad de la persona y puede ser visto como la punta de un iceberg, bajo la cual están diversos factores físicos, emocionales, sociales y espirituales (1). El dolor de difícil control puede deberse a la intensidad, desarrollo de tolerancia a los fármacos, imposibilidad de aumentar la dosis por efectos secundarios, a que posea características especiales que hagan complicado su control, tales como el dolor poco sensible a opioides, el dolor neuropático o el dolor simpático mantenido, el dolor incidental, o aquellos cuadros en que predomina el componente emocional (2). El dolor en los pacientes en cuidado paliativo representa algo más que una sensación nociceptiva e incluye aspectos que tienen que ver con la condición humana, como la personalidad, la afectividad, las emociones, y las relaciones psicosociales (3). En ocasiones este dolor total se convierte en un síntoma de difícil control, a pesar de la intervención adecuada de todo el equipo de cuidados paliativos: psicólogo, asistente social, médicos, enfermeros, y muchas veces se convierte en un síntoma refractario, en el que se debe realizar una sedación paliativa (4).

El caso que se presenta a continuación es el de una mujer, joven, madre de tres niñas, que proviene de un medio socioeconómico deficitario, en la que se diagnostica un dolor total y que debe ser sedada por ser éste refractario a pesar de las intervenciones que consideramos fueron adecuadas.

\section{Descripción del caso}

Paciente de SF, 33 años. Sin antecedentes personales ni familiares previos. Vive con su pareja y tres hijas de 13, 3 años y 18 meses. Recibe pensión por enfermedad. Su pareja, 
desocupado, es el cuidador principal. Recibe ayuda de su madre en el cuidado de sus hijas. Intervenida en enero de 2016 (31 años) por lesión pigmentaria en MID, cursando puerperio de su tercera hija (Hospital Pereira Rossell). La anatomía patológica muestra melanoma maligno de tipo histológico nodular, Breslow $5 \mathrm{~mm}$, Clark IV, con compromiso de márgenes, sin compromiso profundo. Se solicita tomografía axial computarizada (TAC) (16/3/16) que informa: secundarismo encefálico, pulmonar, hepático, óseo, mamario, suprarrenal, peritoneal, inguinal y subcutáneo. Se inicia radioterapia (RT) holocraneana y de columna de T1 a T3 con buena tolerancia. En junio de 2016 comienza primera serie de quimioterapia (QT) con cisplatino, dacarbazina y vinblastina. Se realizan tres series con buena tolerancia. Se realiza tomografía axial computarizada (TAC) de control que evidencia disminución de lesiones encefálicas, hepáticas y pulmonares. Se indican dos series más con buena tolerancia.

En diciembre de 2016 se realiza mastectomía segmentaria bilateral (anatomía patológica: metástasis de melanoma). En enero de 2017 se realiza sexta serie. Se solicita TAC de cráneo y macizo facial por tumoración en maxilar inferior dolorosa que informa lesión lítica de maxilar inferior a izquierda con compromiso de partes blandas. TAC de abdomen y pelvis informa hígado sin lesiones. Lesión lítica de sacro. Se solicita consulta con Radioterapeuta que realiza RT analgésica en maxilar con buen control del dolor. En marzo de 2017 consulta por dolor intenso de columna dorsal que calma parcialmente con AINES. Se ajusta analgesia, se agrega dexametasona vía oral (vo) y se solicita nueva TAC de columna que informa: lesión conocida en T2, lesión lítica a nivel de arco posterior de T10 con compromiso de apófisis espinosa y ambas láminas. Extensa lesión lítica a nivel de alerón sacro. Se realiza nuevamente RT analgésica y posteriormente nueva QT con dacarbazina como monodroga. Luego de ese primer ciclo, plaquetopenia severa. Consulta a las $72 \mathrm{hs}$ por cefalea intensa y tendencia al sueño. Ingresa a Centro de tratamiento intensivo (CTI) por hematoma temporal izquierdo. Buena evolución. Como secuela mantiene paresia de miembro inferior derecho (MID). Al alta (31 de mayo de 2017) es derivada al servicio de cuidados paliativos. Hasta el ingreso al equipo de Cuidados Paliativos, desconocemos que tipo de analgesia recibe y a qué dosis, dado que no contamos con historia clínica previa. Solo contamos con el seguimiento oncológico que refiere "buen" o "mal" control del dolor. Al ingreso al servicio se encontraban los síntomas controlados con Dipirona con codeína (dipirona $500 \mathrm{mg}$ más codeína $10 \mathrm{mg}$ ) cada $8 \mathrm{hs}$, dexametasona $24 \mathrm{mg} /$ día y pregabalina $75 \mathrm{mg} /$ día. Se agrega Lactulosa $10 \mathrm{c}$ cada $12 \mathrm{hs}$, bisacodilo $5 \mathrm{mg}$ cada $8 \mathrm{hs}$ y metoclopramida $10 \mathrm{mg}$ cada $8 \mathrm{hs}$.

El dolor se mantiene controlado por dos meses. Recibe visitas semanales de médico de cuidados paliativos y quincenales por parte de la psicóloga. En esas primeras entrevistas, si bien ella conoce su enfermedad y en ocasiones menciona el pronóstico, prefiere no hablar de ésta (evitación). Se realizó también entrevista con Asistente Social del equipo que se puso en contacto con adscriptos del liceo al que concurre su hija mayor, para que estén enterados de la situación, y con el CAIF (Centro de Atención a la Infancia y la Familia) de la zona. De esta forma se logra que su hija mayor reciba atención por parte del psicólogo del centro educativo y que sus dos hijas menores concurran en doble horario (en general esta modalidad no está permitida en los centros CAIF, pero se realizó el trámite correspondiente y fue aceptada la excepción) y reciban tres comidas en el centro educativo. La paciente presenta hasta ese momento un ECOG 2-3.

Se levanta de la cama al sillón, se higieniza con ayuda, incluso realiza alguna tarea sencilla dentro del hogar. Dos meses después de la primera consulta refiere aumento del dolor en 
MID, tipo neurítico irradiado hasta planta del pie, sin parestesias. Se aumenta analgesia y se indica morfina de liberación inmediata en forma de solución vía oral $10 \mathrm{mg}$ cada $4 \mathrm{hs}$ (60 $\mathrm{mg}$ de morfina oral por día) más rescates (10 mg si dolor) y pregabalina $150 \mathrm{mg}$ en la noche. El resto de la medicación se mantiene igual (laxantes y antieméticos). Mejora el dolor, pero a las $72 \mathrm{hs}$ agrega dolor intenso abdominal, tipo cólico, a predominio de hemiabdomen inferior, que no calma con rescates. Consulta a servicio de urgencia constatando fecaloma, retención aguda de orina (RAO) y se coloca sonda vesical (SV).

Con sospecha de compresión medular se indica internación para estudio, a lo cual la paciente se niega, por no querer dejar a sus hijas. Se consulta con oncólogo tratante sobre posibilidad de RT para control del dolor y tratamiento de la probable compresión, pero está contraindicada por haber recibido ya dosis máximas.

A las $24 \mathrm{hs}$ vuelve a consultar por aumento del dolor, Escala visual analógica del dolor (EVA) 10/10, de similares características. Ingresa a Sanatorio y se comienza con morfina subcutánea (sc), con rápida escalada de dosis, hasta llegar a $160 \mathrm{mg} /$ día en $48 \mathrm{hs}$., en bomba de infusión continua. Se mantienen laxantes y antieméticos y se agregan enemas evacuadores según necesidad. Mal control del dolor, por lo que a las $72 \mathrm{hs}$ se realiza rotación rápida de morfina a metadona sc $30 \mathrm{mg} / \mathrm{dí}^{1}{ }^{1}$, en bomba de infusión continua más adyuvantes, dexametasona $24 \mathrm{mg} \mathrm{sc} /$ día (por otra vía) y pregabalina $75 \mathrm{mg}$ en la mañana y $150 \mathrm{mg}$ en la noche. Se realizan rescates con metadona $5 \mathrm{mg} s \mathrm{sc} /$ dosis y se escala hasta 60 $\mathrm{mg}$ /día en 72 hs., en bomba de infusión continua, más adyuvantes. Se realiza Resonancia magnética (RM) que informa sustitución total del sacro que compromete cola de caballo. Se consulta a terapia del dolor por la posibilidad de medidas intervencionistas para control del dolor, pero son descartadas por el equipo.

Se siguen escalando dosis, sin elementos de intoxicación, pero no se logra control del dolor, EVA 8-9/10. Muy angustiada por dolor y por separación de sus hijas. Se realiza intervención con psicóloga del equipo. De las entrevistas surge un gran componente de angustia por el futuro de sus hijas. Se logra mediante Asistente Social que su marido obtenga la custodia de su hija mayor (fruto de otra pareja), que logra aliviar parte de la angustia. En esta etapa manifiesta claramente conocer el pronóstico a corto plazo, expresando en ocasiones enojo contra la enfermedad. Evita que sus hijas vayan a verla (a pesar de que se habían autorizado las visitas en cualquier horario).

Por falta de stock en farmacia de ampollas de metadona y dado que van disminuyendo las ingestas orales, se debe realizar una nueva rotación a morfina sc. Se realiza rotación rápida de metadona a morfina $400 \mathrm{mg} /$ día sc. en bomba de infusión continua. Se agrega a la bomba midazolam $15 \mathrm{mg} /$ día, metoclopramida $30 \mathrm{mg} /$ día, ranitidina $150 \mathrm{mg} /$ día, y butilbromuro de hioscina $60 \mathrm{mg}$ /día. Se dejan rescates con morfina $10 \mathrm{mg}$ sc si no calma. Por otra vía sc se indica dexametasona $24 \mathrm{mg}$ /día. Se suspende la vía oral. En $48 \mathrm{hs}$ requiere aumentos de dosis por dolor no controlado (hasta 6 rescates diarios), hasta llegar a los $500 \mathrm{mg} \mathrm{sc} /$ día por bomba de infusión continua. En ese momento la paciente comienza con tendencia al sueño, aunque en los momentos de despertar se encuentra lúcida.

El dolor no logra ser controlado a pesar de todas estas intervenciones y continúa con dolor severo (EVA 9/10). Siete días después de la rotación y visto que no se lograba control del dolor se habla con la paciente y su familia y se resuelve con los miembros del equipo

\footnotetext{
${ }^{1}$ DEMO (Dosis Equivalente de Morfina Oral) 320, que haciendo la equivalencia morfina oral: metadona, sería 10:1. Se reduce $30 \%$ de la dosis. En este caso realizamos una reducción menor de la dosis.
} 
iniciar sedación paliativa por dolor total refractario al tratamiento. Se inicia midazolam 30 mg sc /día en infusión continua más rescates de $7.5 \mathrm{mg}$ si Ramsay menor a $3^{2}$. Se escala dosis hasta $90 \mathrm{mg} \mathrm{sc}$. /día. Fallece a las 72 hs.

\section{DISCUSIÓN}

Estudios relativamente antiguos notificaban la incidencia de dolor por cáncer en torno al $50-80 \%$ (5). En comunicaciones más recientes se reportan cifras que son algo inferiores, del $24-60 \%$ en poblaciones en tratamiento activo, y mayor incidencia, del $60-90 \%$, en población con enfermedad avanzada o en fase terminal (6-8). Estas cifras hablan por sí solas de la magnitud del problema, máxime teniendo en cuenta la elevada prevalencia y mejoría en las cifras de supervivencia por cáncer en nuestra época (9).

El alivio sintomático del dolor se puede alcanzar en un buen número de pacientes siguiendo las directrices establecidas en las diferentes guías clínicas, como las de la Organización Mundial de la Salud (OMS) y las elaboradas por diferentes sociedades científicas de cuidados paliativos o de oncología (10). La presencia del dolor y su inadecuado manejo en enfermos con cáncer se ha documentado de manera extensa (11). En 2007 se publicó un metaanálisis que incorporó 52 estudios, con una población representativa de enfermos oncológicos en diferentes situaciones clínicas: después de un tratamiento curativo, durante tratamiento antitumoral, pacientes con enfermedad metastásica y enfermos terminales. En este trabajo se comprobó que la prevalencia es variable en función de la definición empleada de dolor, pero que en cualquier caso es superior al 50\% en todos los tipos de cáncer analizados. En dos revisiones sistemáticas recientes, las cifras de dolor se disparan a rangos del 62-86\% en estadios avanzados de la enfermedad $(12,13)$.

Se constató que los siguientes factores predecían un peor pronóstico en cuanto al alivio del dolor: sufrimiento psicológico intenso, dolor neuropático, dolor episódico, necesidad de aumentar la dosis de opioides varias veces por semana y antecedentes de alcoholismo o de otras drogodependencias. En una serie de casi 300 pacientes, en el $93 \%$ de los que no presentaban ninguna de estas características se obtuvo un control adecuado del dolor, mientras que, en los presentaban una o más de ellas sólo se obtuvo en el $55 \%$, de estos datos surge la clasificación de Edmonton de pronóstico de dolor $(14,15)$.

La aparición de las unidades de terapia del dolor y los servicios de cuidados paliativos en la prestación tanto en el ámbito público como privado han supuesto un importante avance, pero no es suficiente para abarcar todas las necesidades de este grupo de pacientes con sus familias (16). El abordaje del dolor oncológico constituye un reto, desde el punto de vista médico y ético. Los pacientes y con mucha frecuencia los familiares presentan trabas para aceptar el tratamiento con opioides mayores, dado la connotación social del mismo o por identificarlo con la muerte cercana. Existen muchos mitos en cuanto a la utilización de estos medicamentos, pero el más arraigado en la población es que la propia analgesia desencadena la muerte.

Se define dolor total como aquel dolor que se convierte en el centro de la vida de una persona, porque contiene elementos físicos, emocionales, sociales y espirituales (1). La flexibilidad es la llave para el control del dolor en los pacientes, así como los enfermos son

\footnotetext{
2 Escala de Ramsay de sedación: Grado 1: paciente ansioso, agitado. Grado 2: colaborador, orientado. Grado 3 Solo responde a órdenes verbales. Grado 4: Dormido. Responde a algunos estímulos auditivos. Grado 5: Dormido. Solo responde a estímulos táctiles intensos. Grado 6: No hay respuesta.
} 
dinámicos, variando su estadio, respuestas a la terapéutica y preferencias personales, así también debe variar el control del dolor. A pesar de tener una estrategia impecable, con protocolos estandarizados y validados, hay pacientes que no logran calmar el dolor. Hasta un $15 \%$ del dolor oncológico es resistente a morfínicos, precisando para su tratamiento técnicas propias de las unidades del dolor.

La mayoría de los pacientes logran controlar el dolor con las medidas recomendadas por la OMS. Sin embargo, un 10 a $20 \%$ de los pacientes con cáncer avanzado no logran controlar el dolor, ya sea por la fisiopatología de éste o por la incapacidad de absorción o administración de fármacos por la propia enfermedad o por la intolerancia a opioides (17). Se define sedación paliativa terapéutica como "la administración deliberada de fármacos, en las dosis y combinaciones requeridas, para reducir la conciencia de un paciente con enfermedad avanzada o terminal, tanto como sea preciso para aliviar adecuadamente uno o más síntomas refractarios y con su consentimiento explícito, implícito o delegado" (18).

Según la Guía de Sedación Paliativa del PRCPEX, para que la sedación sea considerada ética y legalmente lícita debe reunir determinadas condiciones:

- Debe existir un síntoma refractario.

- Debe dejarse constancia en la historia clínica de la etiología del síntoma, tratamientos instaurados y resistencia a éstos.

- Debe tener como intención aliviar el sufrimiento, con la utilización de drogas adecuadas a dosis adecuadas y proporcionadas al síntoma que queremos aliviar (dosis mínima eficaz).

- Se debe ajustar la dosis y combinación de fármacos a la disminución de la conciencia suficiente para el alivio del síntoma. La monitorización de la respuesta del paciente es fundamental para lograr el alivio.

- Se debe tener siempre el consentimiento informado del paciente. En caso de que éste no pueda dar un consentimiento se puede considerar el expresado por alguien de la familia en quien el paciente haya podido delegar.

- Debe existir evaluación interdisciplinar, proporcionada por profesionales entrenados y siempre bajo su indicación.

No existe evidencia de que la sedación paliativa acorte la vida (19). El término refractario debe ser aplicado a aquellos síntomas que no pueden ser controlados adecuadamente a pesar de los mayores esfuerzos para aplicar tratamientos que no comprometan la conciencia del paciente (20). Los criterios diagnósticos para síntomas refractarios incluyen que el médico debe percibir que los procedimientos invasivos y no invasivos son incapaces de proveer un alivio adecuado, o que están asociados a una morbilidad intolerable para el paciente, o que sea poco probable que mejore los síntomas en un tiempo prudencial. La implicancia de este término es que el dolor puede no ser aliviado en forma adecuada y que en ocasiones la sedación puede ser necesaria para aliviar adecuadamente el síntoma.

En Uruguay existe un protocolo de sedación paliativa en el Manual "Cuidados paliativos. Control de síntomas" del Plan Nacional de Cuidados Paliativos. En éste se define sedación paliativa como "la disminución deliberada del nivel de conciencia mediante la administración de fármacos apropiados con el objetivo de evitar un sufrimiento intenso causado por uno o más síntomas refractarios." Se hace hincapié en que la sedación paliativa es una indicación médica (21). Define síntoma refractario como "aquel que no 
puede ser controlado adecuadamente con los tratamientos disponibles, aplicados por médicos expertos, en un plazo de tiempo razonable y en la fase agónica, breve" (22).

En el caso del dolor, en general éste "está vinculado a destrucción tisular masiva por el tumor, que no puede ser controlado con tratamiento analgésico protocolizado" (22). Para iniciar la sedación paliativa se deben cumplir los siguientes requisitos: que el paciente padezca una enfermedad terminal documentada, que la muerte sea inminente, que exista un síntoma refractario que determine sufrimiento y que se cuente con consentimiento escrito, verbal o delegado a la familia. La sedación debe indicarla el médico tratante, analizarla con el jefe del servicio, asegurar la provisión de medicación e informar al paciente el procedimiento -y/o a la familia $(18,23)$.

La finalidad de este trabajo pretende destacar la importancia de dar un cuidado interdisciplinar, evitando tanto al paciente como a su familia un sufrimiento innecesario, y de la introducción temprana de los cuidados paliativos en pacientes oncológicos, a cualquier edad, en cualquier etapa de la enfermedad y concomitantemente con el tratamiento oncoespecífico. Sabemos que los pacientes jóvenes son derivados más tardíamente a cuidados paliativos y muchas veces reciben tratamientos más agresivos, aunque sean fútiles (24).

El carácter intolerable y refractario al tratamiento de esta situación obliga en muchas ocasiones al equipo asistencial a indicar la sedación del enfermo. En líneas generales, una buena muerte es la muerte sin dolor y sin otros síntomas mal controlados, que no se prolonga de manera artificial, con la compañía de la familia y los amigos, con la posibilidad de haber sido informado adecuadamente sobre la enfermedad, participando en la toma de decisiones, eligiendo dónde se desea morir (domicilio u hospital) y contando en cada caso con el apoyo sanitario adecuado (25). La sedación paliativa no es eutanasia y las diferencias están en el objetivo, el proceso y el resultado (23).

El caso tratado se trató de una paciente altamente compleja por su situación clínica, la cual ingresó al servicio en forma tardía luego de una complicación neurológica. Si bien sabemos que la introducción temprana al equipo de cuidados paliativos no cambia el pronóstico de la paciente, está comprobado que mejora la calidad de vida. En todo el tiempo que estuvo atendida hizo uso de su autonomía, definiendo qué es lo que era beneficioso para ella y su familia. Se la trató en equipo multidisciplinario atendiendo todas las necesidades. Centraremos los siguientes párrafos en el síntoma, su tratamiento y el recorrido hasta la sedación.

Las causas de sedación descritas en la literatura son delirio, disnea, dolor, hemorragia, náuseas - vómitos, y psicológicas. Broeckaert en Alemania en el año 2000 obtuvo los siguientes resultados como causales de sedación: 39\% delirio, 38\% disnea, $22 \%$ dolor, $8,5 \%$ hemorragia, $6 \%$ náuseas y vómitos y $21 \%$ psicológicas (26). En el año 2008 la unidad de cuidados paliativos del Hospital Central de las fuerzas Armadas, en Montevideo, Uruguay encontró que los causales de sedación fueron $60 \%$ delirio, $17 \%$ disnea, $45 \%$ dolor, $0 \%$ hemorragia, $2 \%$ náuseas y vómitos y $0 \%$ psicológicas (23).

La escalada analgésica siguió los protocolos de la OMS, llegando al tercer escalón con el uso de opioides fuertes y coadyuvantes, necesitando aumentar rápidamente las dosis y la rotación a opioides de mayor potencia con menos efectos indeseables, dado que el síntoma no podía ser controlado. Se realizó trabajo interdisciplinario con psicóloga y trabajadora 
social, ya que se trataba de un dolor total, que debía ser abordado desde todas las áreas. En este punto del dolor oncológico refractario, se pensó en tratamiento invasivo consultando a los anestesistas de terapia de dolor, pero fue descartado por la paciente ya que no quería nuevas terapias intervencionistas y que, dada la extensión de la lesión y que la paciente había recibido altas dosis de RT, éstas no eran posibles.

En este momento con una expectativa de vida muy corta y un síntoma refractario, se planteó la sedación paliativa a la paciente y su familia, que fue consentido en forma verbal por la paciente (que permanecía lúcida) y fue aceptado por su madre y su pareja. Fue indicado por dos de los médicos tratantes, que conocían a la paciente y su familia desde hacía varios meses y tratado en la coordinación del equipo de cuidados paliativos de la institución.

Se inició sedación con Midazolam sin retirar los fármacos analgésicos que venía recibiendo por vía parenteral. Se reunieron entonces los requisitos básicos para iniciar la sedación: la paciente era portadora de una enfermedad oncológica avanzada, en etapa terminal. Tenía un síntoma refractario (el dolor total). Se obtuvo el consentimiento verbal por parte de la paciente y la sedación fue indicada por el médico tratante en consenso con el resto del equipo.

\section{CONCLUSIONES}

Si bien el dolor es un síntoma común en los pacientes con enfermedad oncológica avanzada y en la mayoría de los casos logramos controlarlo, hay un porcentaje de pacientes que no responden o responden mal a la terapéutica habitual.

Debemos tener presente siempre en nuestros pacientes aquellos factores personales y de la patología que nos puede indicar que estaremos frente a un paciente con un dolor de difícil control. Tener presente estos factores de riesgo y saber con los recursos que contamos puede ser determinante para definir a un dolor difícil como refractario. Este dolor puede tener, además del componente físico, un componente psicológico, social, espiritual, que lo convierten en ocasiones en un dolor total, que debe ser abordado por todos los miembros del equipo. Este dolor total también puede ser refractario al tratamiento. Si se cumplen todos los pasos diagnósticos y terapéuticos $\mathrm{y}$ aplicamos las guías nacionales $\mathrm{e}$ internacionales para sedación paliativa, debemos tener presente que el dolor total también puede ser causa de sedación paliativa y constituye un escalón terapéutico más para aliviar un síntoma.

\section{Agradecimientos}

A K.F. y su familia que tanto nos enseñó en el transcurso de su enfermedad. Fue un honor acompañar el recorrido.

\section{REFERENCIAS BIBLIOGRÁFICAS}

1 López-Sánchez JR, Rivera-Largacha S. Historia del concepto de dolor total y reflexiones sobre la humanización de la atención a pacientes terminales. Rev Cienc Salud. 2018;16(2):340-356.

2 Paredes, M. Qué hacer ante un dolor de difícil y rebelde. Paliativos sin Fronteras. Internet 2010. (Consultado 12 de julio de 2018) Disponible en: http://paliativossinfronteras.org/wp-content/uploads/Dolor-dif\%C3\%ADcil-Marin.pdf 
3 Krikorian, A. \& Limonero, J. T. (2015). Factores asociados a la experiencia de sufrimiento en pacientes con cáncer avanzado. Avances en Psicología Latinoamericana, $33(3), 423-438$.

4 A.S. Strömgren,M. Groenvold,M.A. Petersen,D. Goldschmidt,L. Pedersen,M. Spile. Pain characteristics and treatment outcome for advanced cancer patients during the first week of specialized palliative care, J Pain Symptom Manage., 27 (2004), pp. 104-113

5 R.G. Twycross. The terminal care of patients with lung cáncer, Postgrad Med J., 49 (1973), pp. 732-737.

6 Van den Beuken M.H. Everdingen,J.M. De Rijke,A.G. Kessels,H.C. Schouten,M. Van Kleef,J. Prevalence of pain in patients with cancer: A systematic review of the past 40 years, Ann Oncol., 18 (2007), pp. 1437-1449.

7 Di Maio,C. Gridelli,C. Gallo,L. Manzione,L. Brancaccio, S. Barbera. Prevalence and management of pain in italian patients with advanced non-small-cell lung cancer $\mathrm{Br} \mathbf{J}$ Cancer., 90 (2004), pp. 2288-2296.

8 A.S. Strömgren,M. Groenvold,M.A. Petersen,D. Goldschmidt,L. Pedersen,M. Spile. Pain characteristics and treatment outcome for advanced cancer patients during the first week of specialized palliative care, J Pain Symptom Manage., 27 (2004), pp. 104-113.

9 C.E. DeSantis,C.C. Lin,A.B. Mariotto,R.L. Siegel,K.D. Stein,J.L. Kramer. Cancer treatment and survivorship statistics, 2014, CA Cancer J Clin., 64 (2014), pp. 252-271.

10 S. Mercadante,F. Fulfaro, World health organization guidelines for cancer pain: A reappraisal, Ann Oncol., (2005), pp. v132-v135.

11 H. Breivik,N. Cherny,B. Collett,F. De Conno,M. Filbet,A.J. Foubert.

Cancer-related pain: A pan-european survey of prevalence, treatment, and patient attitudes, Ann Oncol., 20 (2009), pp. 1420-1433.

12 M.H. Van den Beuken-van Everdingen,J.M. De Rijke,A.G. Kessels,H.C. Schouten, M. Van Kleef,J. Patijn. Prevalence of pain in patients with cancer: A systematic review of the past 40 years Ann Oncol., 18 (2007), pp. 1437-1449.

13 S.C. Teunissen, W. Wesker,C. Kruitwagen,H.C. De Haes,E.E. Voest,A. De Graeff. Symptom prevalence in patients with incurable cancer: A systematic review. J Pain Symptom Manage., 34 (2007), pp. 94-104.

14 Bruera E, Schoeller T, Wenk R, MacEachern T, Marcelino S, Hanson J, et al. A prospective multicenter assessment of the Edmonton Staging System for cancer pain. J Pain Symptom Manage 1995; 10:348-355.

15 Bruera E, Kuehn N, Miller MJ, Selmser P, Macmillan K. The Edmonton Symptom Assessment System (ESAS): a simple method for the assessment of palliative care patients. J Palliat Care. 1991 Summer;7(2):6-9.

16 World Health Organization. Cáncer pain relief. 2nd. ed. Geneva: World Health Organization, 1996. 
17 McHugh ME1, Miller-Saultz D, Wuhrman E, Kosharskyy B. Interventional pain management in the palliative care patient. 2013. International Journal of Palliative NursingVol. 18, No. 9.

18 Sánchez, M.A. Cuervo, M.A, Sanchez, R. Varillas, M.P., et all. Guía clínica. Sedación paliativa del PRCPEX. Secpal. (consultado julio de 2018) disponible en: http://www.secpal.com/\%5CDocumentos\%5CBlog\%5Cguia-de-sedacion.pdf

19 Casas-Martínez ML, Mora-Magaña I. ¿La sedación paliativa acorta la vida de los pacientes? pers.bioét. 2017; 21(2): 204-218.

20 Cherny N. I., on behalf of the ESMO Guidelines Working Group. ESMO Clinical Practice Guidelines for the management of refractory symptoms at the end of life and the use of palliative sedation. Annals of Oncology, Volume 25, Issue suppl_3, 1 September 2014, Pages iii143-iii152.

21 Gómez Sancho, M. Ojeda Martin, M. Cuidados Paliativos. Control de síntomas. Las Palmas, gran Canaria. Hospital universitario de gran Canaria. 2009. (consultado julio de 2018) 47(2) se consigue en:

https://www.cgcom.es/sites/default/files/Cuidados\%20paliativos.\%20control\%20de\%20si ntomas.

22 Cuadernos de la Fundació Víctor Grífols i Lucas Ética y Sedación al final de la vida. N. ${ }^{\circ} 9$ - (2003) 9(2).

23 Santos D, Della Valle A, Barlocco B, Pereyra J, Bonilla D. Sedación paliativa: experiencia en una unidad de cuidados paliativos de Montevideo. Rev. Méd. Urug. (Internet). Junio 2009 (consultado diciembre 2018) ; 25( 2 ): 78-83.

24 Snaman J. M., Kaye E. C., Baker. N. Jessie J. Palliative Care Involvement Is Associated with Less Intensive End-of-Life Care in Adolescent and Young Adult Oncology Patients. Journal of Palliative Medicine. Volume 20, Number 5, 2017.

25 González Barón, M. Gómez, C. Vilches, Y. Última etapa de la enfermedad neoplásica progresiva: Cuidados en la agonía, síntomas refractarios y sedación. Medicina Clínica. Volumen 122. Issue 11. Septiembre 2006. Pág 421- 428.

26 Broeckaert B. Palliative sedation define or why and when terminal sedation is not eutanasia. Abstract, 1 st Congress RDPC, December 2000, Berlín, Alemania. 